\title{
Der sächsische Konferenzminister Gottlob Adolf Ernst von Nostitz und Jänckendorf (1765-1836)
}

\section{Ein Beitrag zum 250. Geburtstag}

\author{
Boris Böhm
}

\section{Stationen seines Lebens und Schaffens}

Gottlob Adolf Ernst von Nostitz und Jänckendorf wurde am 21. April 1765 als Spross des alten Oberlausitzer Adelsgeschlechtes auf Gut See bei Niesky geboren. Sein Vater starb bereits 1768, und die Familie übersiedelte nach Oppach, wo sie im herrschaftlichen Niederhof lebte. Die Verantwortung für den begabten Jungen lag seitdem bei der Mutter, die ihm im eigenen Haus eine sorgfältige Erziehung und umfassende humanistische Bildung zuteil werden ließ. Mit 15 Jahren begann er zu dichten und behielt sich diese Leidenschaft ein Leben lang.

Bereits im 16. Lebensjahr immatrikulierte sich Nostitz an der Universität Leipzig. Er studierte zwei Jahre mit äußerster Intensität Staats-, Verwaltungs- und Rechtswissenschaften und legte 1783 ein hervorragendes Examen ab. Anschließend begann er seine berufliche Laufbahn als Auditor (Prüfer) beim Oberhofgericht in Leipzig. Im Februar 1785 trat er als Finanzrat beim kurfürstlichen Geheimen Finanzkollegium in Dresden in den sächsischen Staatsdienst ein.

Am 31. Mai 1786 heiratete Nostitz in Schkeuditz die 17jährige Henriette Sophie von Bose (1769-1848). Aus der Ehe gingen acht Töchter und vier Söhne hervor. Nachdem er mündig geworden war, übernahm er 1786 das väterliche Rittergut Oppach mit Wurbis, das er ein halbes Jahrhundert bis zu seinem Tode ungeachtet der zahlreichen sonst von ihm ausgeübten Ämter bewirtschaftete.
Im Jahre 1792 übernahm er in seiner Oberlausitzer Heimat ein wichtiges ständisches Amt: ihm wurde vom Oberlausitzer Provinziallandtag das Amt eines Landesältesten des Bautzner Kreises übertragen. Dies war eine einflussreiche Stellung, da die Oberlausitzer Stände auch nach dem Übergang an den Kurfürsten von Sachsen im Prager Frieden von 1635 zahlreiche Sonderrechte behielten. Wie bereits zuvor in seinem Ehrenamt als Beisitzer des Landständischen Waisenamtes zeigte sich in dieser Funktion früh das Engagement von Nostitz für die soziale Fürsorge. Zuerst setzte er von ihm als notwendig erachtete Reformen auf sozialem Gebiet auf seinen Gütern, er besaß zeitweilig auch die Güter Jenkwitz und Doberschau, um. So errichtete er 1794 in Oppach ein Armenhaus. 1801 veröffentlichte er in Görlitz einen „Versuch über Armenversorgungsanstalten in Dörfern, in näherer Beziehung auf das Markgrafthum Oberlausitz. " Damit seiner Zeit voraus, sind die vom ihm aufgestellten Grundsätze 40 Jahre später in die sächsische Armenordnung vom 23. Oktober 1840 eingegangen. Die Hofdienste seiner Untertanen in Oppach wandelte Nostitz in geringe Geldabgaben um. Dem Schulwesen galt sein besonderes Augenmerk. Auch seine Verpflichtungen als Gerichtsherr in Oppach nahm er ernst. Auferlegte Strafgelder überwies er häufig zu Gunsten des von ihm begründeten Armenhauses. Am Neujahrtag 1802 ließ er der Oppacher Gemeinde von der Kanzel abkündigen: „...Ihr wißt, daß mir euer Wohlstand und die Ruhe und Zufriedenheit jedes Einzelnen unter euch auch in der Entfernung, zu welcher mich meine Geschäfte zwin- 
gen, am Herzen liegt, und daß es mir gewiß angenehm ist, euch nützlich zu werden..."

Nostitz wohnte mit seiner Familie von 1790 bis 1806 überwiegend in Bautzen, wo 1791 auch sein Sohn Eduard Gottlob geboren wurde, der als sein prominentestes Kind von 1836 bis 1844 das Amt des sächsischen Innenministers ausübte. Sein Sohn Julius Gottlob (1797-1870) war von 1840 bis 1864 sächsischer Gesandter beim Deutschen Bund, die Tochter Clotilde Septimia (1801-1852) trat als Dichterin in die Fußstapfen ihres Vaters.

Nostitz starke geistigen und literarischen Interessen führten ihn 1790 als Mitglied in die Oberlausitzische Gesellschaft der Wissenschaften, die damals 57 Mitglieder zählte. Im Alter von 30 Jahren wurde er 1795 zum Präsidenten dieser Gesellschaft gewählt. Während seiner bis 1817 andauernden Präsidentschaft hatte er bedeutenden Anteil an der Entfaltung einer regen wissenschaftlichen Tätigkeit. Der vielseitig interessierte, gelehrsame und redegewandte Präsident erwarb sich durch bemerkenswerte Ansprachen und sein integratives Wirken viele Sympathien inner- und außerhalb der Gesellschaft.

Der 1802 vom Oberlausitzer Provinziallandtag als Amtshauptmann bestimmte Nostitz übernahm im Juni 1804 als Oberamtshauptmann die Spitze der in Bautzen ansässigen Provinzregierung für die Oberlausitz. Dazu hatte er sich den Oberlausitzer Ständen durch die umsichtige Bekleidung bisheriger parlamentarischer Ämter, sein ausgleichendes Wesen und diplomatisches Geschick empfohlen. Bereits im September 1807 berief der sächsische König Friedrich August I. Nostitz als Oberkonsistorialpräsidenten nach Dresden, wohin er auch seinen Wohnsitz verlegte. Hier wirkte er unter anderem an der Überarbeitung der Verfassung der Universität Leipzig mit.

Am 13. April 1809 erfolgte durch den sächsischen König die Ernennung von Nostitz zum Konferenzminister und wirklichen Geheimen Rat. Als einer von drei Konferenzministern gehörte er zu den ranghöchsten Staatsmännern des Königreiches. In dieser Funktion war Nostitz bis zum Jahre 1831 Mitglied des Geheimen Konsiliums - des obersten Beratungsorgans des Königs - und seit 1817 eines von insgesamt sieben Mitgliedern des Geheimen Rates, dessen Vorsitz er in den letzten Jahren inne hatte. Weiterhin wurde ihm vom König im Mai 1809 die Leitung der Königlichen Kommission für die Landes-Straf- und Versorgungsanstalten übertragen, die er bis 1831 ausübte.
Der selbst dichtende Nostitz trat zeitlebens als ein Gönner der Künste auf. Nach der Napoleonzeit war er eine prägende Gestalt des „Dresdner Liederkreises“ und erlangte unter dem Pseudonym Arthur vom Nordstern mit einigen seiner Werke eine gewisse Popularität. Weiterhin gilt er auch als einer der ersten Übersetzer von Byrons Werken ins Deutsche. Zu seinem Freundeskreis zählten der Maler Gerhard von Kügelgen und der Altertumswissenschaftler Karl August Böttiger.

Als eifriger Freimaurer, deren Tugenden Humanität und Toleranz er verkörperte, nahm er in dem Bund eine hohe Stellung ein und übte von 1827 bis 1836 das Amt des Landesgroßmeisters aus. Bemerkenswert ist auch sein Einsatz für die Gründung des Sächsischen Altertumsvereins.

In den krisengeschüttelten Jahren 1830/1831 wirkte Nostitz als Staatsminister im neuen Kabinett von Bernhard von Lindenau an der

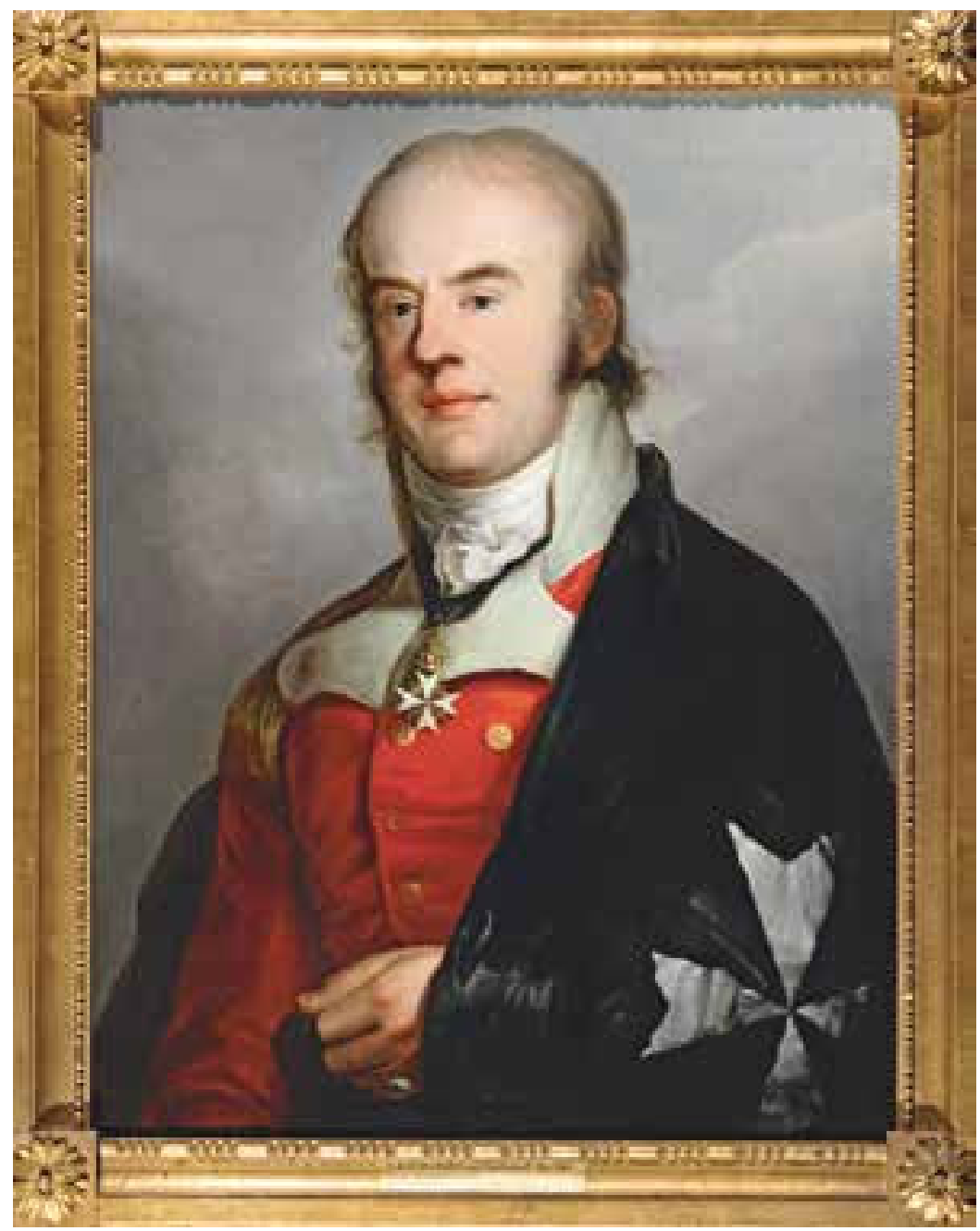

Unbekannter Maler, Porträt Gottlob Adolf Ernst von Nostitz und Jänckendorf, um 1800, Öl auf Leinwand

(c) Kulturhistorisches Museum Görlitz

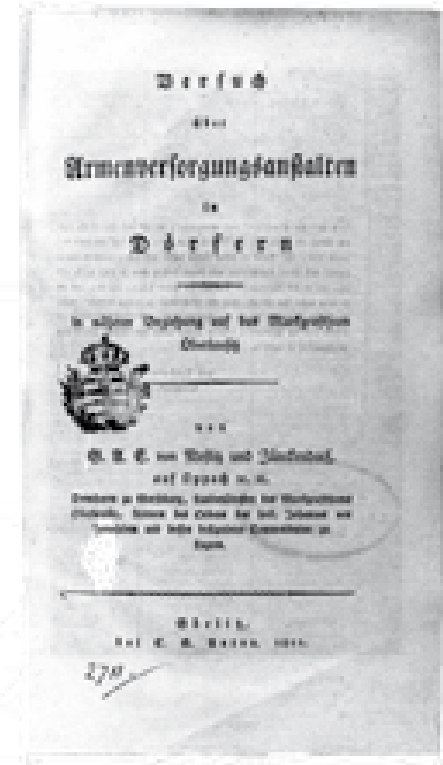

Titelblatt einer von Nostitz 1801 veröffentlichten sozialen Schrift (c) SLUB Dresden 
Auszug aus dem Erlass von König Friedrich August I. vom 17. Mai 1809, betreffend die Ernennung von Gottlob Adolf Ernst von Nostitz und Jänckendorf zum Direktor der Kommission für die Straf- und Versorgungsanstalten (C) Sächsisches Staatsarchiv, Hauptstaatsarchiv Dresden

Titelseite der berühmten Publikation von Nostitz über den Sonnenstein, 1829 (c) Bibliothek des Sächsischen Krankenhauses Arnsdorf

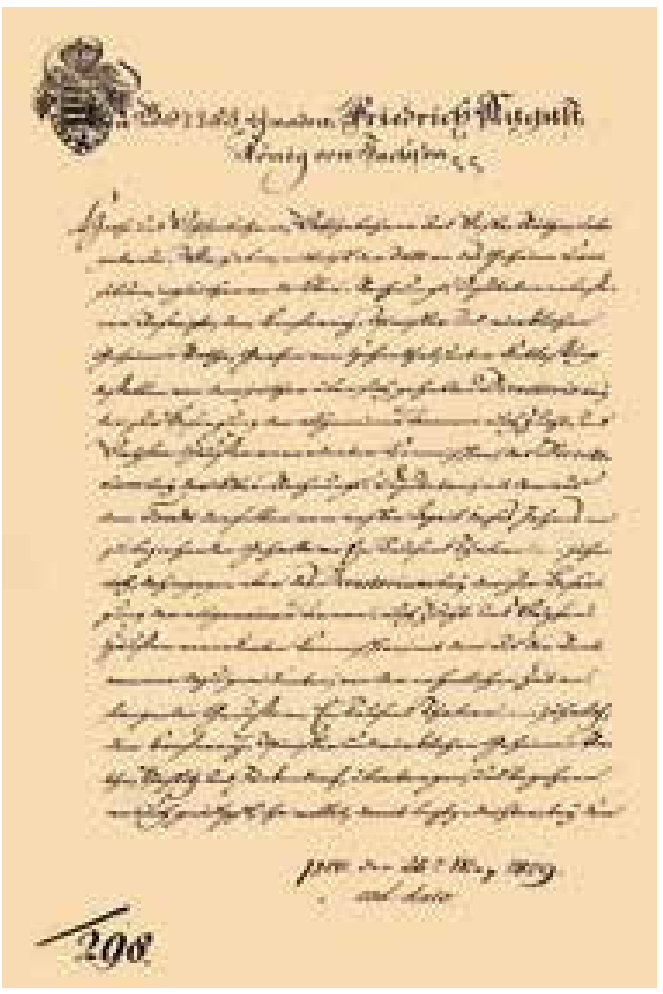

Erarbeitung einer zeitgemäßen liberalen sächsischen Landesverfassung mit, die er vor Inkrafttreten am 4. September 1831 kontrasignierte. Ende 1831 trat er in den Ruhestand und widmete sich fortan auf seinem Oppacher Gut ganz seiner Familie und seinen literarischen Neigungen. Kurz vor seinem Tod

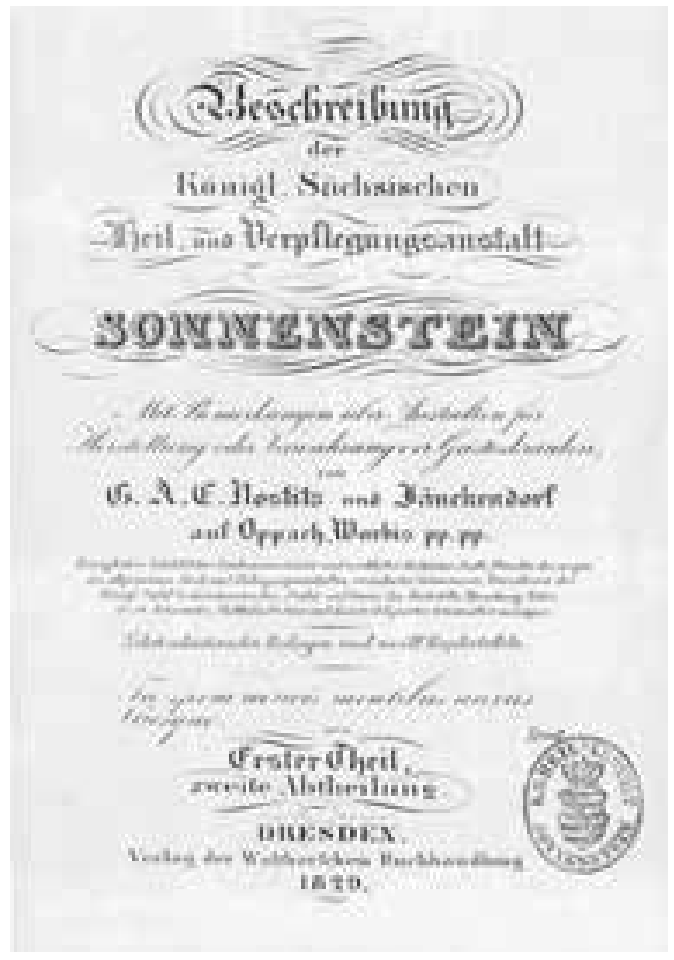

verlieh ihm die Philosophische Fakultät der Universität Leipzig die Ehrendoktorwürde. Am 15. Oktober 1836 verstarb Gottlob Adolf Ernst von Nostitz und Jänckendorf 71-jährig in Oppach, wo er auch begraben wurde.

\section{Direktor der Kommission für die Straf- und Versorgungsanstalten}

Im historischen Rückblick ist Nostitz' langjährige Tätigkeit als Direktor der „zur Besorgung der allgemeinen Armen-, auch Zucht- und Waisenhäuser verordneten Commission" von besonderer Bedeutung, zu dem er am 17. Mai 1809 durch den sächsischen König ernannt worden war.

Die von Nostitz angestrebte Reformierung der Landesanstalten hatte das Ziel der Überwindung des undifferenzierten Typs des Internierungshauses. Nostitz ließ sich von den Ideen der Aufklärung und Menschenrechtsforderungen im Gefolge der Französischen Revolution leiten, berücksichtigte aber auch Sachzwänge wie die ständige Überfüllung der sächsischen Anstalten. Als Direktor hatte Nostitz Verantwortung für die Organisation und Ausgestaltung des Anstaltswesens sowie für die wirtschaftlichen Belange und Finanzierung der Einrichtungen. So hatte er auch die Oberaufsicht über die Landeslotterie, deren Einnahmen für die Finanzierung der Landesanstalten verwendet wurden. $\mathrm{Zu}$ seinem Verantwortungsbereich gehörten 1809 zunächst die Strafund Versorgungsanstalt in Waldheim, die Armen- und Waisenanstalt Torgau, die Zuchtanstalt Torgau und die Strafanstalt Zwickau. Die Humanisierung des Strafvollzuges in Zwickau und Waldheim war ihm ein wichtiges Anliegen.

Nachdem im November 1810 auf Forderung Napoleons der sächsische König die Neubefestigung Torgaus angeordnet hatte, stellte Nostitz den Antrag zur Gründung einer Heilanstalt auf dem Sonnenstein. Bereits am 8. Juli 1811 konnte diese dann in den Gebäuden der ehemaligen Landesfestung eröffnet werden. Sie war von Beginn an das "Lieblingskind“ von Nostitz. Er sah hier die Chance, ein seinen philanthropischen Neigungen entsprechendes staatliches Musterinstitut $\mathrm{zu}$ formen, das sich ausdrücklich der Heilung von Kranken und nicht nur deren Verwahrung zum Ziel setzte. Besondere Bedeutung maß er der humanen Behandlung der 200 hier aufgenommenen psychisch kranken Menschen bei. Er beschränkte die Patientenzahl, um angesichts eines relativ kleinen Beamtenkörpers therapeutische Zielsetzungen nicht zu gefährden. Auf ein hohes 
Niveau der Ernährung, Bekleidung und Unterkunft legte er auch bei den nicht selbstzahlenden Patienten großen Wert. Als Direktor der Kommission hatte er sich auch mit der Rechtmäßigkeit ärztlicher Vorstellungen, insbesondere zur Dauer der Unterbringung der Patienten, auseinanderzusetzen.

Besondere Verdienste erwarb er sich im Herbst 1813 bei der Versorgung und Rettung aller Insassen während der Kämpfe um Dresden und der Fremdnutzung der Anstalten Sonnenstein und Waldheim. Er sorgte maßgeblich für die Bereitstellung der Mittel für den Wiederaufbau des schwer beschädigten Sonnensteins. Große Schwierigkeiten hatten Nostitz und die von ihm geleitete Kommission nach 1815 durch die Abtretung von fast 60 Prozent des sächsischen Territoriums $\mathrm{zu}$ überwinden. Sachsen verlor dadurch die Strafanstalt Lichtenburg, die 1812 auf Initiative von Nostitz eingerichtete Irrenanstalt Sorau und das Waisenhaus Langendorf. Nostitz befasste sich mehrere Jahre mit den bei der Abtretung zu beachtenden Grundsätzen und rang um die Aufteilung von Vermögen und Kapitalbeständen der abgetretenen Einrichtungen.

Eine notwendige Veränderung betraf die staatliche Fürsorge für Waisenkinder, von denen nicht alle in städtischen Asylen und Waisenversorgungshäusern untergebracht werden konnten. Der Initiative von Nostitz war 1824 die Errichtung einer Landeswaisenanstalt für 80 Kinder auf dem Rittergut Bräunsdorf bei Freiberg zu danken. In dieser Anstalt genossen die unter anderem aus Waldheim überführten Waisenkinder fortan Erziehung und Ausbildung.

Nicht nur unter Ärzten und Philanthropen, auch in Regierungskreisen erlangte der Sonnenstein den Ruf als Musteranstalt. So erhielten Nostitz und seine Kommission den Auftrag, den Höfen in Wien und Paris sowie der russischen Kaisermutter Maria Fjodorowna Berichte über diese Anstalt zu erstatten. Die Bitte letzterer war der Anlass, dass Nostitz 1829 seine berühmte „Beschreibung der Königl. Sächsischen Heilund Verpflegungsanstalt Sonnenstein" erarbeitete. Darin untersuchte Nostitz die gesamte theoretische und praktische Psychiatrie seines Zeitalters, erörterte die Einrichtung und Organisation der Irrenanstalten und vermittelte in Auswertung seiner 20-jährigen Erfahrungen auf diesem Gebiet bedeutsame Richtlinien und Regulative für die Ausgestaltung des Sonnensteins.

1827 fasste die Kommission unter Nostitz den Beschluss, in Waldheim die unheilbar Geisteskranken von den Strafgefangenen zu trennen und Schloss Colditz für erstere einzurich-

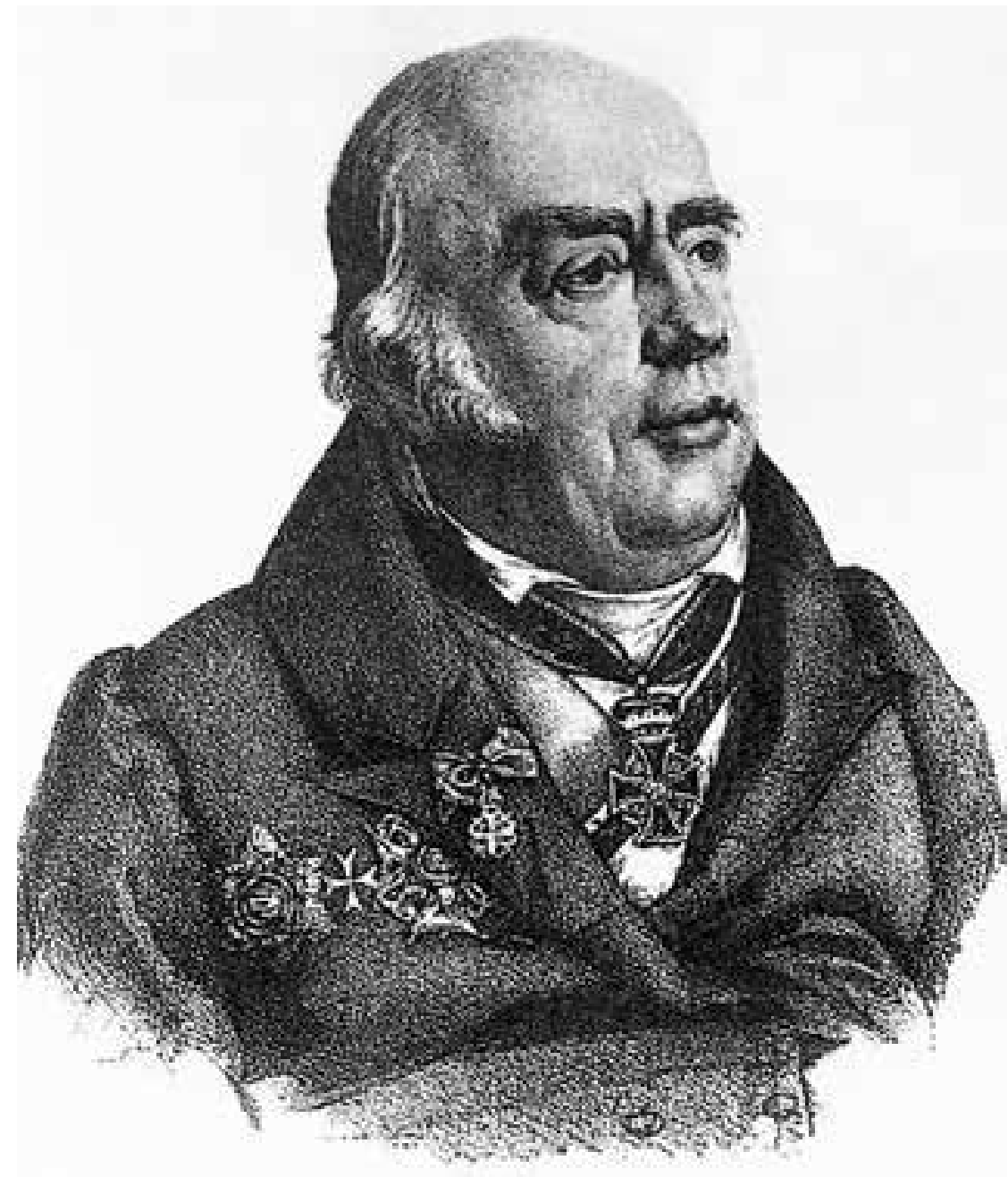

ten. Dies geschah aus der Erkenntnis, dass sich die Doppelfunktion von Verwahr- und Versorgungsanstalt einerseits und Strafanstalt andererseits als nicht praktikabel erwiesen hatte. Nostitz weihte am 12. November 1829 die Versorgungsanstalt Colditz ein, deren Leitung dem von ihm geschätzten Arzt Dr. Hayner übertragen wurde. Die Profilierung von Colditz als mit der Heilanstalt Sonnenstein korrespondierende Pflegeanstalt vollendete die 1811 unter Führung von Nostitz begonnene Entmischung der undifferenzierten Zucht-, Waisen- und Armenhäuser in Torgau und Waldheim. Waldheim diente fortan als reine Strafanstalt.

1831 gab Nostitz mit dem Ministeramt auch den Direktorenposten auf, in dem er sich enorme Verdienste um das sächsische Gesundheitsund Sozialwesen erworben hatte. Sein soziales Empfinden und philanthropisches Handeln hatten ihn immer wieder besonders für die Kranken und Armen sorgen lassen. Sein Interesse an den Problemen Geisteskranker war unter den deutschen Staatsmännern der 1. Hälfte des 19. Jahrhunderts einzigartig.
Unbekannter Zeichner, Altersbildnis von Gottlob Adolf Ernst von Nostitz und Jänckendorf, Lithografie, 1836

\section{Autor \\ Dr. Boris Böhm \\ Gedenkstätte \\ Pirna-Sonnenstein \\ Schlosspark 11 \\ 01796 Pirna}

